seems the best time to look for that planet. It is then at a considerable altitude directly above the setting sun, but the small size of the spot marked 5the spots are graded for relative brightness-indicates that the planet may not be very bright on those evenings. Venus will evidently be a magnificent object in March, over the south-west horizon and about halfway up to the zenith. The curve for Mars shows a singular loop in the autumn, and during August, September and October the planet is actually farther east each successive evening at the time of sunset. This loop, by the way, is not to be thought of as only the ordinary loop made relative to the background of stars, but rather as a loop made relative to the leafless branches of the old tree at the south end of my garden, expressed-over a short range at least-by an equation of the type

$$
\log _{\varepsilon} \eta=A+\frac{B}{T} \quad . \quad . \quad .
$$

'Taking therefore Dr. Newton Friend's equation for latent heat and casting it in the form

$$
\text { Molar } L=4 R \frac{T_{2}-T_{2}}{T_{1} T_{2}} \log _{\varepsilon} \frac{\eta_{1}}{\eta_{2}}
$$

we get by inserting equation (1)

Molar $L=4 R \frac{T_{1}-T_{2}}{T_{1} T_{2}} B\left(\frac{1}{T_{1}}-\frac{1}{T_{2}}\right)=4 R B \ldots$

which looks simple enough.

Taking the viscosity results on helium liquid I obtained in Toronto and plotting them over the range $3 \cdot 5^{\circ}-4 \cdot 2^{\circ} \mathrm{K}$. (I restrict myself to temperatures not far from the boiling point, $4 \cdot 2^{\circ} \mathrm{K}$.), I find :

$$
\log _{\varepsilon} \eta=A+\frac{9}{T}
$$

There is no point at present in evaluating $A$. Substitution in equation (3) gives a value for the molar $L$ as 72 calories. This is equivalent to 18 calories per gm.

If, however, we use the data obtained at Leyden, the latent heat of vaporization of liquid helium 1 is of the order of $\mathbf{5}$ calories per gm. (Reference to the Leyden entropy diagram of helium shows an increase of entropy of $1 \cdot 2$ units per gm. when liquid helium 1 evapor. ates at $4 \cdot 2^{\circ} \mathrm{K}$, and $1 \cdot 2 \times 4.2=8$ calories very nearly).

Therefore whether we consider Dr. Newton Friend's result or the value I quote above as being more correctly obtained by his equation, we see that the agreement with the correct value is not good. Hence his equation must be in error.

JoHN SATTERLY.

Department of Physies,

University, Toronto.

Jan. 19.

the track of an equatorial star at sixteen hourly intervals, and some planetary positions are marked below the eastern horizon. Jupiter and Saturn are nearly as sedate as fixed stars.

About the middle of January the planets Venus and Saturn appeared close together, and the same will be true of Venus and Mercury in April. Mars and Jupiter are to be so close together at the end of October that the one spot numbered 11 is allowed to mark the position of both.

London.

S. L. WALKDEN.

Feb. 2.

\section{Latent Heat of Evaporation of Liquid Helium}

THE letter of Dr. J. Newton Friend in NATURE of December 26, 1936, on this subject raises interest in his equation. I have tested it with the following results :

As many know, the variation of the coefficient of viscosity of a liquid with temperature can often be
I AM grateful to Prof. Satterly for directing atten. tion to the discrepancy. Accepting $5 \times 4=20$ as the molar latent heat of evaporation of helium, the value $31 \cdot 3$ calculated with the aid of the ClausiusClapeyron equation is certainly wide of the mark; so is 36.2 which I calculated from my equation using Prof. Burton's data. But as my equation was derived on the assumption that the Clausius-Clapeyron equation holds, clearly if the latter fails the former may be expected to do so also.

Both equations have, however, been found to give very satisfactory results with a large number of substances. Assuming the vapour pressure and viscosity data for liquid helium are correct, instead of suggesting be better to suggest that helium is abnormal ? That such may well be the case is evidenced by the curious fact that liquid helium II is much more fluid than that the equations "must be in error", would it not 